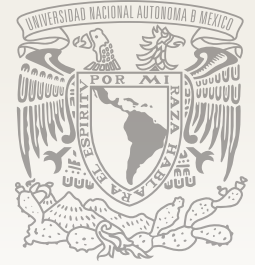

\title{
Revista
}

Pueblos y fronteras

\section{LAS ORGANIZACIONES DE PRODUCTORES DE CAÑA Y SUS RELACIONES DE PODER. EL CASO DE LA ASOCIACIÓN LOCAL DE CAÑEROS DE CASASANO, EN CUAUTLA DE MORELOS, MÉXICO}

Luis Enrique Parral Quintero

parralq.luise@hotmail.es

Universidad Autónoma Metropolitana-Unidad Iztapalapa

\section{RESUMEN}

El cultivo de caña de azúcar forma parte de una tradición agrícola establecida en la época colonial. Es ese tiempo la hacienda se encargaba de organizar su producción. Esto ha ido cambiando. Con el movimiento revolucionario se resquebrajó la estructura hacendaria y surgieron nuevos actores en el campo, llamados ejidatarios. Al mismo tiempo se establecieron decretos encaminados a proteger los complejos industriales para que los ejidatarios aledaños al ingenio continuaran con su producción. Ante este panorama es importante analizar la forma en la que los productores se han ido articulando y las relaciones de poder que se generan en este proceso mediante la intervención de las asociaciones locales de productores.

Palabras clave: Productores de caña, asociaciones de productores, agricultura por contrato, relaciones de poder.

\author{
SUGARCANE PRODUCER ORGANIZATIONS AND THEIR POWER RELATIONS: THE CASE \\ OF THE CASASANO LOCAL SUGARCANE FARMERS ASSOCIATION IN CUAUTLA DE MORELOS, MEXICO
}

\section{ABSTRACT}

Sugarcane cultivation is part of an agricultural tradition established during the colonial period. During that time, sugarcane production was organized by large land holdings known as haciendas. This has changed. With the revolutionary movement, this landed estate structure crumbled and rural areas experienced the emergence of new communal landholders, known as ejidatarios. At the same time, laws aimed at protecting industrial complexes were decreed enabling the communal landholders adjacent to sugar refineries to continue with production. Within this scenario, it is important to analyze the way in which producers have been articulating as well as the power relationships generated through this process via the intervention of local producer associations.

Key words: Sugarcane producers, producer associations, contract farming, power relationships. 


\section{INTRODUCCIÓN}

Las características del clima y la localización geográfica del estado de Morelos han sido de vital importancia para el desarrollo de la actividad cañera. En ese suelo se practica esta actividad desde que el conquistador Hernán Cortés se establece en lo que hoy es el municipio de Cuernavaca, y estuvo ligada a la consolidación de varias haciendas azucareras y trapiches a lo largo y ancho de la entidad.

La producción de caña de azúcar se practica en el ejido de Casasano en Cuautla, Morelos, por lo menos desde el siglo Xvi. En la actualidad se realiza bajo un modelo de agricultura por contrato; es decir, para desarrollar dicha actividad productiva los cañeros deben celebrar un contrato con el Fideicomiso Ingenio Casasano «La Abeja», ya que de esta manera van a conseguir vender la cosecha de la vara dulce. Para que el contrato pueda realizarse el ingenio pide a los cañeros que se afilien a alguna de las asociaciones de productores que se encuentran en su zona de abastecimiento, tal como lo establece la Ley de Desarrollo Sustentable de la Caña de Azúcar.

Las asociaciones locales de productores de caña forman parte de confederaciones nacionales como la Campesina (CNC) y la de Propietarios Rurales (CNPR); a nivel local las asociaciones actúan como intermediarias entre los productores y el ingenio azucarero; se encargan de organizar, junto con el personal del ingenio, los ciclos de la zafra (cosecha de la caña de azúcar). Dan apoyo logístico con la finalidad de hacer eficiente el periodo de cosecha y aprovechar el tiempo (comprende cinco a seis meses: comienza en noviembre o diciembre y culmina en abril o mayo) y los recursos humanos disponibles para dicha actividad.

Este tipo de asociaciones de productores también se encargan de gestionar recursos económicos, materiales y mano de obra, es decir, consiguen a los jornaleros (mano de obra estacional) y la maquinaria que será empleada para realizar el corte, alce y acarreo hasta el patio del ingenio. En tiempo de siembra proporcionan capacitación a los cañeros para que conozcan las variedades de caña, fertilizantes y otras formas de cultivar que pudieran resultar rentables tanto para los productores como para el ingenio, y de esta forma ambos pueden obtener mayores ganancias y los rendimientos de la caña de azúcar a la hora de extraer el jugo dulce que de ella emana.

Es de vital importancia, entonces, analizar cómo se relacionan los productores de caña con la Asociación Local de Cañeros de Casasano CNPR (ALCC-CNPR de aquí en adelante) en dicho ejido, ya que, como se ha mencionado, la relación obedece a un modelo de agricultura por contrato, y es escenario de relaciones de poder que solo pueden reconocerse si se observa detalladamente, ya que se consolidan compromisos entre los diferentes actores sociales que participan en la producción de caña de azúcar.

\section{ACTORES SOCIALES EN LA PRODUCCIÓN DE CAÑA DE AZÚCAR: CONCEPTOS CLAVE}

Desde la antropología política y particularmente desde el enfoque turneriano (procesualista) se realiza un análisis sobre la forma en que se establecen las relaciones de poder en la ALCC-CNPR; por tal motivo es relevante recuperar la definición de lo político analizado como un proceso, bajo la idea procesualista que propone Abélès (1997) ya que considera que lo político son aquellos procesos que se originan por la elección y realización de objetivos; esto quiere decir que para el desarrollo de los procesos políticos existe una combinación de tres elementos en una misma dinámica: el poder, la determinación y la realización de objetivos públicos o privados. Se ha recurrido a este enfoque porque destaca que el poder se ejerce en todos los puntos en los que se dan relaciones sociales y permea el espacio social por completo. Busca espacios concretos en eventos críticos, puntos en los que se despliega estratégicamente; busca describir y analizar en los dramas sociales las formas múltiples de su ejercicio. En este sentido cabe aclarar que los dramas sociales que menciona Turner permiten describir y analizar el carácter multidimensional de la vida, así como las contradicciones estructurales, las inconsistencia y los conflictos entre las normas, los grupos, hombres, mujeres y las diferentes interpretaciones que todo conflicto 
promueve, la forma en que se expresan las continuidades y cambios en las relaciones de poder, el poder de los símbolos y los símbolos del poder, los modos en que se ventilan los intereses divergentes (Díaz 2014b).

La definición de poder que propone el enfoque procesualista permite analizar la forma en que se desarrollan las relaciones en la producción de caña de azúcar. La intervención de la ALCC-CNPR es un reflejo de la realización de los objetivos públicos, ya que los cañeros buscan obtener ganancias por sus cosechas, y por esa razón se asocian con quienes pretenden representar sus intereses generales.

El análisis procesual, de acuerdo con Díaz (2014b: 56), permite crear «puentes de análisis de las estructuras, los procesos y la agencia humana, entre las sociedad y la intención, entre la cultura y la subjetividad» ya que el análisis implica, en palabras de Castro y Rodríguez (2009), explicar de manera profunda la resolución de conflictos, la toma de decisiones y la negociación de disputas que surgen en torno a alcanzar metas de carácter público en las sociedades humanas. En el análisis procesual las áreas funcionales del análisis de procesos son tres: la primera es el régimen, o también conocido como las «reglas del juego», y consiste en acuerdos que regulan la forma en la cual las demandas se sitúan en el sistema, se realiza la negociación y se toma una decisión con efecto entre los grupos; la segunda es «el gobierno»: una serie de estatus interconectados y cuyos roles se insertan en la toma de decisiones políticas, y está formado por las instituciones de la organización administrativa; la tercera área funcional es la de los políticos «oficiales»: aquellos actores que se dedican a disputar el poder.

\section{LA CAÑA DE AZÚCAR EN MORELOS}

La consolidación de la actividad cañera en Morelos se debió a las ventajas naturales que presentan los valles de Cuernavaca y Cuautla, ya que ofrecían a los inversionistas grandes incentivos económicos relacionados con la producción y distribución de mercancías como azúcar y aguardiente, además de que permitían que se ejerciera un gran poder y control político sobre los habitantes (Mentz 1984).
Stavenhaguen (1974) menciona que con la reforma agraria que se inicia después de la revolución mexicana se transforma totalmente la estructura agraria del país; con esta reforma se busca distribuir la propiedad de la tierra a todos aquellos que habían sido despojados durante la colonia; desaparecen los grandes latifundios y dan paso a la aparición de nuevos actores sociales en el campo.

Se establece el ejido como una nueva forma de tenencia de la tierra. En principio la da en posesión pero no en propiedad a las comunidades de agricultores. Esto quiere decir que los miembros de estas entidades agrarias tienen el derecho de cultivar individualmente una parcela dada de tierras cultivables.

Pese a que con la reforma agraria se buscaba eliminar los grandes latifundios, el gobierno federal crea zonas de abastecimiento para los ingenios para que pudiera seguirse desarrollando la actividad productiva; con estos decretos se pretendía intensificarla y de ese modo cubrir las necesidades del mercado interno y a largo plazo generar excedentes hacia el mercado internacional (Gildo 2002).

La configuración de la industria azucarera en Morelos muestra cómo se gestan procesos en los que hay una lucha por recursos y cómo la actividad cañera responde a un proceso de comercialización más amplio; se puede observar que hay una puesta en escena de las reglas del juego que se establecen para desarrollar la actividad productiva, donde las condiciones geográficas y climatológicas son un detonante que adquiere importancia, ya que eran las idóneas para practicar el cultivo de caña de azúcar.

El desarrollo histórico es parte de ese fluir de la vida social que menciona Turner. En su curso se muestran procesos de transición en la forma en que se practica la actividad productiva, se muestran procesos sociales, transiciones que alteran, modifican y transforman la existencia (Díaz 2014a).

\section{CONFIGURACIÓN DEL CORPORATIVISMO EN MÉXICO}

El campo mexicano no es solamente un espacio agrario delimitado y diferenciado del es- 
pacio urbano; es un área rural en que se desarrolla una amplia gama de relaciones entre los diversos grupos sociales que lo conforman, que abarcan desde productores agropecuarios, con diferentes tipos de campesinos, pasando por trabajadores a domicilio del sector informal, asalariados locales y migrantes que se vinculan tanto al campo como a la ciudad; en estos procesos se gestan intereses económicos y políticos que determinan la forma en que cada uno de los actores habrá de articularse al campo (Carton de Grammont 1996a).

Después del proceso de la revolución mexicana se comienza con la restauración del orden. En este caso, junto a la figura de los ejidatarios (con el reparto agrario) se consolidan estructuras políticas, medios de organización de los ejidatarios que dan origen a las organizaciones corporativas.

La estructura corporativa es, en la actualidad, el medio por el que se incorporan los productores de caña en la actividad agroindustrial, ya que para incorporarse a esta actividad tienen que formar parte de una asociación de productores que represente sus intereses generales frente al ingenio.

El carácter político del campesinado en México está representado por organizaciones corporativas que se gestaron durante el periodo cardenista. Es el caso de la Confederación Nacional Campesina (CNC) y de la Confederación Nacional de Productores Rurales (CNPR), esta última consolidada en 1946. Ambas instituciones, durante el siglo xx, controlaban la representación popular del sector campesino y negociaban con el Estado. Sin embargo, a finales del mismo siglo la relación entre dichas confederaciones y el sistema político del Estado se transformaron profundamente debido al nuevo modelo económico que se instauró hacia 1990 (Carton de Grammont 2003).

La estructura corporativa que se encuentra presente en el Estado mexicano forma parte de un proceso que se constituye en tres momentos clave:

El primero tiene origen en los años posteriores al periodo revolucionario. Los grupos militares porfiristas habían sido derrotados y buscaban reorganizarse política y socialmente para opo- nerse a cualquier proyecto de reparto agrario. Se promulga la Ley de Asociaciones Agrícolas de 1932, cuya función era reemplazar las cámaras agrícolas que se oponían a los planteamientos del movimiento revolucionario.

El segundo corresponde al cardenismo. Se configura el Partido de la Revolución Mexicana y se crea la Confederación Nacional Campesina (CNC), en 1937, organización concebida como un instrumento social necesario para consolidar la reforma agraria.

El tercero tiene origen en la coyuntura de la creación del Partido Revolucionario Institucional (PRI) y de la Confederación Nacional de la Pequeña Propiedad (CNPP) en 1946 (Carton de Grammont 1996a).

La configuración de estas dos organizaciones corporativas muestra un esquema organizativo y complejo en ambas estructuras agrarias cuya función representa intereses particulares y colectivos tanto de la figura ejidal ( $\mathrm{CNC}$ ) como del sector privado (CNPR); en dichas estructuras políticas se desarrollan procesos de negociación desde el Estado hacia las bases de la organización formadas por los productores (Carton de Grammont 1996a).

Pero durante la década de los setenta, la relación entre el Estado y los agricultores se torna difícil, ya que se presentan dos rupturas entre las organizaciones corporativas y el Estado. La primera se gesta de 1975 a 1976, cuando el movimiento agrarista afectó a los llamados «neolatifundios» (Carton de Grammont 1996b: 23) que se ubicaban en las mejores tierras del país, principalmente en el noreste. En este proceso los empresarios y el gobierno, que en ese entonces era representado por el presidente Luis Echeverría, tuvieron discrepancias que culminaron en la creación de un nuevo organismo de representación de los agricultores privados, en diciembre de 1975, no afiliado al partido oficial: la Unión Agrícola Nacional (UNAN).

La segunda ruptura que se gestó entre las organizaciones y el Estado se gesta con la nacionalización de la banca en 1982. Este proceso afectó la cúpula empresarial del campo, ya que había intereses en instituciones bancarias regionales, aunque cabe señalar que esta crisis de confianza se subsanó durante el mandato de Miguel de la 
Madrid, ya que era su interés terminar con el reparto agrario de tal manera que no se afectara la legislación (Carton de Grammont 1996b).

Antes de la aplicación del modelo neoliberal existía una diferencia legal en la tenencia de la tierra, una clara dicotomía entre el sector ejidal y el de la pequeña propiedad. Esta diferenciación acentuaba los procesos organizativos de los productores, incluidos los que solicitaban tierra.

Carton de Grammont (1996a) considera que esa diferencia entre sectores se ha ido diluyendo por la nueva estructura agraria que se mantiene en el campo, constituida por cuatro elementos básicos: la extinción del ejido como la única forma de organizar la producción ejidal; la importancia que han adquirido las cadenas productivas para organizar la producción comercial, dado que se ponen en juego intereses de los agentes económicos (productores, comerciantes, industriales) que intervienen en dichas cadenas. El tercer elemento se refiere a la existencia de pobladores que no pueden reproducirse a partir de la relación con la producción agropecuaria, sino por la que desarrollan con el sector informal (talleres de manufactura en poblados rurales que en ocasiones elaboran artesanías para el mercado nacional o internacional, o trabajan como maquiladores del sector industrial); finalmente, el cuarto elemento se refiere a la migración y los flujos de dinero desde las ciudades del interior del país o en otros casos desde el extranjero, con la finalidad de reproducir la unidad familiar campesina.

Los elementos anteriores moldean las nuevas relaciones que se establecen en el campo mexicano. Esto no quiere decir que haya desaparecido el corporativismo. Plantean un esquema organizativo más complejo que la «doble estructura piramidal» (Carton de Grammont 1996a: 12), en donde la primer estructura se refiere al sector de la CNC y la segunda al sector privado mediante la CNPP y las asociaciones de productores. Dichos esquemas permitían regular los procesos de negociación desde la parte estatal hasta las bases que se encuentran formadas por los productores.

La aplicación de la política neoliberal provoca una división en el campo. El concepto de «productor» se restringe a aquellos capaces de competir en el mercado abierto, los otros, que son considerados como «pobres», quedan fuera de la política productivista y se ubican en las encaminadas a combatir la pobreza (Carton de Grammont 1996a).

Bajo este esquema de reorganización de la producción y restructuración del campesinado el mismo Carton de Grammont (1996a) afirma que las organizaciones corporativas se han modificado y han diversificado sus objetivos en cuatro tipos de organizaciones con finalidades distintas:

Gremial o de representación social: se define por los actores sociales en torno a demandas generales. En este tipo de organización se presenta una participación política activa, tal es el caso de la CNC, que se definirá por su participación política en las filas del partido de Estado. Este tipo de organizaciones también pueden inhibir su participación política con la finalidad de lograr mayor consenso en torno a las demandas sociales que defienden.

Económica para la producción: abarca desde la compra de insumos hasta la obtención del financiamiento. Este tipo de organización se define por agentes económicos, ya sean individuales o colectivos, con intereses productivos específicos; sus demandas tienen que ver con lo que «debe ser el campo»: expresan los intereses de los pequeños productores pero no tienen una definición política.

Económica para el consumo: se significa por actores sociales organizados en torno a la problemática del bienestar familiar. En general defiende las demandas específicas con un alto grado de politización, ya que vela por los intereses de los grupos más pobres que no encuentran alternativas en ningún espacio de la sociedad.

Étnica: representada por actores sociales con un amplio espectro de demandas, que pueden ir desde las de corte cultural, económico, territorial, laboral, como en los casos del Consejo Estatal de Organizaciones Independientes Campesinas (CEOIC) y de las redes étnicas oaxaqueñas, hasta organizaciones que suponen definiciones políticas nacionales, como el Ejercito Zapatista de Liberación Nacional (EZLN). 


\section{EL INGENIO DE CASASANO Y LA ASOCIACIÓN LOCAL DE CAÑEROS}

El ingenio de Casasano se conformó como ejido en 1929; es resultado del reparto agrario posrevolucionario; en ese año se dota a la congregación de Casasano con 310 hectáreas, 66 áreas de riego y 103 hectáreas de temporal, y suma un total de 413 hectáreas y 66 áreas. En la actualidad dicho ejido está conformado por un total de 155 ejidatarios, y los usos que se le dan a la tierra son el cultivo de plantas ornamentales, caña de azúcar, maíz y para la construcción (Parral 2011b).

El municipio de Cuautla, ubicado en el estado de Morelos, está conformado por varios ejidos, de entre ellos destaca el de Casasano porque en él se halla el ingenio La Abeja. El ejido colinda con los de Cuautla, Cuautlixco, Eusebio Jáuregui, El Hospital, Calderón y Tetelcingo; y con la pequeña propiedad de Casasano.

El abastecimiento hídrico en Casasano es mediante canales de riego alimentados por el manantial Las Tazas, del ejido de Cuautlixco. Estos canales irrigan las parcelas en que se cultiva la caña de azúcar. Los canales llevan por nombre Casasano Grande, Sauce Chino y Ahuehuepan, y en su mayoría están revestidos de cemento. En el primer canal el agua se obtiene a partir de bombeo en el manantial antedicho, mientras que en los otros dos canales el riego se da por escurrimiento.

En Casasano hay dos asociaciones de productores de caña, ambas encargadas de representar los intereses generales de los productores de la zona de abastecimiento frente al ingenio azucarero de Casasano La Abeja. Dicho ingenio forma parte del consorcio Fondo de Empresas Expropiadas del Sector Azucarero (FEESA) y mantiene una producción total de $27.15 \%$ del azúcar en México, y a nivel nacional posee el sexto lugar en rendimiento de caña de azúcar. Durante la zafra del año 2008-2009 recibió 369316.160 toneladas de caña en bruto, las cuales fueron procesadas.

\section{LA CNPR Y LA ALCC}

La Confederación Nacional de la Pequeña Propiedad Agrícola se funda en 1946 en el marco de una reorganización del partido oficial, que en ese entonces era promovida por el presidente Miguel Alemán. Más tarde, en 1971 cambió su nombre por el de Confederación Nacional de la Pequeña Propiedad (CNPP).

En el campo que representa la industria azucarera de Casasano, Morelos, la ALCG-CNPR forma parte de la Confederación Nacional de Productores Rurales (CNPR) y de la Confederación Iberoamericana de Productores de Caña de Azúcar (CIPCA). Este esquema está presente en 15 estados del país, por medio de siete uniones estatales, 56 asociaciones locales y por el Comité Ejecutivo Nacional. Para la producción de caña de azúcar la Confederación Nacional de Productores Rurales (CNPR) divide el territorio nacional en tres regiones: Centro-Huasteca, Occidente-Sureste y Golfo. El caso que nos ocupa, el ejido de Casasano, forma parte de la región cañera Centro-Huasteca, conformada por los ingenios de Atencingo, Calípam, Emiliano Zapata y Casasano La Abeja, este último ubicado al oriente del estado de Morelos (Parral 2011a).

La ALCC-CNPR se establece en el ejido de Casasano durante la década de 1980. En sus inicios se encontraba integrada por un grupo de aproximadamente 80 personas afiliadas; se mantiene con este número de productores aproximadamente una década. Durante ese periodo se establece de manera precaria. Por un tiempo comparte oficinas con la Unión Local de Productores de Caña (UNLP-CNC), asociación local adscrita a la Confederación Nacional Campesina. En 2007, a partir de créditos que le facilita la CNPR a nivel nacional, la ALCC-CNPR consigue los recursos económicos necesarios para establecer oficinas propias y de esta manera aumentar la calidad en la asesoría para los productores de caña.

En los últimos años, la ALCC-CNPR ha incrementado el número de sus agremiados, esto debido a que, de acuerdo con los representantes de dicha organización cañera, les ofrece mayores ganancias que la ULPC-CNC.

En este escenario existe, por otro lado, cierto tipo de actores sociales que simplemente prefieren quedarse en la asociación a la que han pertenecido toda su vida; estos consideran que los productores (ejidatarios) que cambian de 
asociación que represente sus intereses son «los arrimados o los arrepentidos»; mencionan que no deberían de estar incorporados en la ALCCCNPR porque esta es sólo para los pequeños propietarios, y argumentan que los ejidatarios deben estar en la CNC.

La configuración de la industria azucarera se ha transformado: pasó de ser un modelo de producción organizado por la hacienda, a otro que responde a las reglas del juego del capital económico mundial, en que la producción se articula por contrato, con base en el financiamiento, ya sea de una parte o de la totalidad del proceso, y de ese modo los productores se quedan sometidos o se comprometen a vender el total de su producción a la empresa que proporciona dicho financiamiento, a un precio que se fija mediante un contrato, previo a la producción.

Este tipo de contratos comprometen la entrega de las cosechas en el patio (batey) del ingenio, y los dueños asumen la obligación del pago siempre y cuando se haya firmado previamente un contrato, al inicio de la siembra, y para asegurarse de que así será, los ingenieros realizan recorridos de supervisión para tomar medidas; esto significa que si los cañeros no pueden organizar de ese modo su actividad y vender en esos términos su cosecha, correrán el riesgo de quedarse con el producto al final de la zafra. Bajo este formato, algunos de los productores consideran vulnerada su libertad.

Para cumplir con el ritual que implica formar parte de la agricultura por contrato, deben además afiliarse a organizaciones de productores (de carácter corporativo). Para ello deben llenar algunos requisitos, como tener copia de sus escrituras, en caso de ser pequeño propietario, y si es ejidatario, el certificado de la parcela, una constancia del ejido al que pertenece, que especifique dónde tiene su siembra y si el productor es ejidatario o arrendador, y en caso de que esté realizando un cambio de asociación, presentar una carta de renuncia a la organización en la que se encuentren afiliados.

Una vez articulados al proceso productivo de la caña de azúcar, los productores realizan la siembra entre septiembre y diciembre, y en casos extremos hasta enero, porque si no lo hacen así no será factible que el ingenio les haga un contrato y les corte la caña, puesto que el ciclo es por periodos de 12 a 14 meses (en la plantilla). El cultivo tarda hasta 14 meses para llegar a condiciones de corte, y si se siembra en febrero, el corte de la caña sería en los mes de mayo, y para ese entonces es cuando la zafra finaliza, y no es posible que el ingenio corte el producto.

Los dirigentes de la Asociación Local de Cañeros de Casasano adquieren el compromiso de representar a los productores de caña dentro de un marco ético y legal, así como de ver por los intereses de cada uno de sus agremiados. De acuerdo con los representantes, la asociación se enfoca en el desarrollo y el aprovechamiento de los recursos que permiten consolidar la agroindustria cañera en Casasano.

El modelo de agricultura por contrato requiere, entonces, que los productores se organicen, y esa exigencia se ve materializada en la Asociación Local de Cañeros CNPR, que se encarga de las labores que van desde conseguir la mano de obra y maquinaria necesaria para realizar el corte de la caña, hasta la supervisión y la logística; esto significa que organiza y supervisa el corte y traslado hasta el patio del ingenio; durante el cultivo y el crecimiento se encarga de dar seguimiento hasta el punto de madurez, y una vez que esto sucede avisa a los productores cuando la caña ya es susceptible de corte; una vez realizado el corte y acarreo de la caña, se encarga de informar a los productores sobre los tiempos de pago: preliquidación y liquidación final.

En lo que respecta a las labores de logística, las asociaciones de productores consiguen al personal que realizará el corte de caña, que por lo general son jornaleros (mano de obra estacional) de los estados de Guerrero, Puebla, Oaxaca y en ocasiones de Chiapas; además, se encarga de proveer las herramientas de trabajo: al inicio de la zafra le entrega a cada cortador un petate, un machete y una lima; además, cada cortador debe presentarse para que la asociación gestione su alta en el seguro social; esto relacionado con el corte manual. En el corte mecanizado la asociación consigue las cosechadoras y alzadoras, que son rentadas a productores del mismo ejido; los dueños se encargan del mantenimiento. Lo que concierne al corte, levantamiento y 
acarreo de la caña lo gestiona la secretaria de cosecha.

Una de las tareas que ha realizado la asociación recientemente es la creación de albergues para los cortadores de caña; uno de ellos fue inaugurado el 17 de noviembre del 2010. Para su construcción se recibió apoyo del comité nacional, así como de los productores y de los camioneros; el edificio contaba con una primera planta, con 23 cuartos, pero está proyectada para 50 habitaciones. El inmueble albergará a 100 personas, dos por cuarto. Y se cuenta con otro inmueble para los familiares de los cortadores, que en ocasiones los acompañan.

En el esquema de agricultura por contrato, este tipo de asociaciones desempeña el papel de intermediario entre el productor y el ingenio. El tiempo de zafra suele der de 165 días, aproximadamente; esto incluye tiempo perdido al comienzo de la zafra, que se refiere al plazo en que el ingenio realiza pruebas para observar el funcionamiento de la maquinaria y ver que no haya algún tipo de inconveniente que retrase la molienda; por lo general, en el ingenio se lleva una bitácora para observar el progreso del funcionamiento, en ocasiones pueden pasar de 10 a 30 días antes de que se comience a moler la caña.

\section{EL CRISTAL PROCESUALISTA EN LA ORGANIZACIÓN CAÑERA}

La mirada diacrónica ha permitido conocer en qué forma surge y se constituye la actividad productiva de la caña de azúcar en Morelos, principalmente en el caso de Casasano; muestra los diversos actores y las transformaciones que sufren, así como las reglas del juego bajo las cuales se rige el esquema productivo en diferentes momentos históricos. Muestra a los actores y los recursos que involucra esta actividad; la apropiación de recursos por parte de los españoles consolida un esquema productivo, que a lo largo de 500 años puede considerarse tradicional.

La rápida vista histórica permite observar y reconocer lo que la propuesta turneriana nos recuerda: la vida fluyen, ninguna sociedad es estática y lo que vemos en la actualidad forma parte de procesos de transformación.

$\mathrm{El}$ alcance de objetivos forma parte de las relaciones de poder en la industria del azúcar, y al mismo tiempo moldea la forma en la que la sociedad se reorganiza; en este sentido se puede afirmar que la apropiación de recursos forma parte del alcance de metas de determinado grupo social y esto conlleva al establecimiento de arenas de lucha, donde los actores que forman parte de este campo van a perseguir sus objetivos.

En este sentido, el análisis procesualista (o turneriano) tiene pertinencia porque pese que se habla de una actividad agroindustrial y de campesinos, en tiempo de zafra es cuando se puede observar la mayor concurrencia de personas que se insertan en la actividad, ya sea como cañeros o como trabajadores directos o indirectos del ingenio; por tanto, se puede considerar que es una actividad que afecta a un grupo de sociedades.

Con la producción de caña de azúcar se puede observar un control de los recursos naturales, agua y tierra, ya que para el cultivo de la vara es necesario acaparar aquellas franjas que sean susceptibles de irrigación. El control en el riego va a permitir que se obtengan rendimientos elevados a la hora de procesarse el producto, y eso impactará en forma positiva o negativa en los pagos.

En este proceso, que tiene que ver con el alcance y la satisfacción de objetivos públicos, es importante la comunicación entre individuos y grupos; por tal motivo, hay que recuperar el carácter humanístico para asumir que en los dramas sociales se movilizan razones, intereses, voluntades, deseos, fantasías, emociones, aspiraciones (Díaz 2014b).

El contrato que se establece en la producción de caña de azúcar se convierte en una puerta o alternativa para que los cañeros puedan alcanzar sus metas colectivas, que de forma inmediata serían el desarrollo de su actividad y la comercialización de su cosecha a un precio determinado, a diferencia de los cultivos de hortalizas que se encuentran «desprotegidos» (las comillas son mías), pese a que los ejidatarios y pequeños pro- 
pietarios deban ajustarse a las necesidades y controles de calidad, tiempos de siembra y cosecha.

El papel de los representantes de la Asociación Local de Cañeros de Casasano se ubica en el campo de la agricultura por contrato; las actividades y los compromisos de esta organización se vincula tanto con los productores de caña como con los dueños del ingenio. Se despliegan, asimismo, relaciones de poder, ya que los cañeros, al aceptar las reglas buscan alcanzar objetivos particulares (la subsistencia), pero al mismo tiempo pretenden acceder a objetivos públicos, que son de interés para la comunidad cañera: obtener seguridad en la venta de sus cosechas.

Por otro lado, la ALCG-CNPR procura el cumplimiento de las condiciones que establece el contrato que de forma individual celebra cada productor de caña con los representantes del ingenio azucarero; en este sentido, la toma de decisiones y la negociación frente al complejo agroindustrial corre a cargo de este actor social, que de esta manera facilita el proceso de negociación y realiza la función de interlocutor.

\section{CONCLUSIONES}

El caso expuesto ha sido abordado desde la teoría procesualista, porque permite entender cómo se establecen las relaciones de poder entre los actores sociales que pertenecen al campo de la industria azucarera que se practica en Casasano, Morelos; se ha realizado una descripción diacrónica considerando la forma en la que se establecen los cultivos de caña de azúcar en Morelos, ya que la introducción de un nuevo modelo productivo genera transformaciones en la organización social, política y económica de una sociedad.

Cabe mencionar que para que pueda funcionar esta agroindustria se necesitan recursos naturales, como grandes extensiones de tierra y agua para irrigar los cultivos; en este sentido la lógica de la desaparecida hacienda podría seguir siendo la misma: sembrar en grandes extensiones para producir azúcar, pero con la variante de que los que realizan dicha actividad son los cañeros, ya sean ejidatarios, pequeños propietarios o simplemente arrendatarios. En este sentido es importante recuperar la defini- ción propuesta de poder que aporta el enfoque procesualista, ya que los recursos naturales son escasos, tienen límites y no son infinitos.

Para finalizar, es importante señalar que el modelo de agricultura por contrato deja en un espacio liminar a los encargados de producir la caña de azúcar, ya que pese a que se incorporan a este modelo productivo en el campo (en términos turnerianos) de la agroindustria del azúcar, no pueden ser considerados como obreros, pues son trabajadores de los ingenios; pero tampoco son campesinos que se dediquen a cultivar hortalizas, o produzcan para el autoabasto; son simplemente cañeros que buscan subsistir.

\section{FUENTES DE CONSULTA}

Abélès, Marc, 1997, «La antropología política: nuevos objetivos, nuevos objetos», Revista Internacional de Ciencias Sociales, 153, en www.unesco.org/ issj/rics153/abelespa.html, [consultado: 27 de noviembre de 2012].

Bartra, Armando, 1996, «La agroexportación del sureste, Suburbio tropical de las metrópolis económicas», El México Bárbaro, plantaciones y monterías del Sureste durante el porfiriato, México, El Atajo, pp. 11-33.

Carton de Grammont, Huber, 2003, «Las organizaciones campesinas en la transición democrática», ponencia presentada en el $I V$ Congreso $A M E R, 20$ al 23 de junio, Morelia.

_ $1996 a$, «Introducción» en Hubert Carton de Grammont (coord.), Neoliberalismo y organización en el campo mexicano, México, UNAM/Plaza y Valdés, pp. 9-20.

—, $1996 b$, «La organización gremial de los agricultores frente a los procesos de globalización en la agricultura", en H. Carton de Grammont (coord.), Neoliberalismo y organización en el campo mexicano, México, Universidad Nacional Autónoma de México, Plaza y Valdés, pp. 21-68

Castro, Pablo y Luis Rodríguez, 2009, «Antropología de los procesos políticos y del poder», Alteridades, 38, pp. 107-127.

Cheryl, Martin, 1984, «Historia Social del Morelos Colonial», en Horacio Crespo (coord.), Morelos cinco siglos de historia regional, México, UAEM, pp. 81-93.

Chihu, Aquiles, 1996, «El procesualismo simbólico. Una propuesta de análisis en la cultura política», Polis, 97, pp. 175-196. 
Díaz, Rodrigo, 2014a, «Del análisis situacional a los dramas sociales» en R. Díaz, Los lugares de lo politico, los desplazamientos del símbolo. Poder y simbolismo en la obra de Victor W. Turner, México, UAM / Gedisa, pp. 29-70.

, 2014b, «Los lugares de lo político. Una relectura de la antropología procesualista» en R. Díaz, Los lugares de lo político, los desplazamientos del símbolo. Poder y simbolismo en la obra de Victor W. Turner, México, UAM / Gedisa, pp. 71-108.

Gildo, Pedro, 2002, «La industrialización de la caña y el desarrollo sustentable en el noreste del estado de Colima: 1950-2000», tesis de maestría inédita, México, Universidad de Colima.

Melville, Robert, 1979, Crecimiento y Rebelión. El desarrollo económico de las haciendas azucareras en Morelos (1880-1910), México, Nueva Imagen, Centro de Investigaciones del Desarrollo Rural.

Mentz, Brígida, 1984, «La región morelense en la primera mitad del siglo xIx: Fuentes e hipótesis de trabajo", en Horacio Crespo (coord.), Morelos cinco siglos de historia regional, México, UAEM, pp. 131-147.
Parral, Luis, 2011a, «La organización de productores cañeros en Casasano; ventajas y contradicciones», ponencia presentada en el VIII Congreso de la Asociación Mexicana de Estudios Rurales, 24 al 27 de mayo, Puebla.

— $2011 b$, «La Asociación Local de cañeros y el Ingenio de Casasano, una relación necesaria en Casasano», tesis de maestría inédita, México, Universidad Autónoma del Estado de Morelos.

Stavenhaguen, Rodolfo, 1974, «La economía de plantación» en R. Stavenhaguen, Las clases sociales en las sociedades agrarias, México, Siglo XXI Editores, pp. 79-108.

Turner, Victor, 1974, «Dramas, fields, and metaphors. Symbolic action in human society», trad. Carlos Reynoso, Londres-Ithaca, Cornell University Press.
Fecha de recepción: 23 de enero de 2014

Fecha de aceptación: 30 de septiembre de 2014 